\section{Relation between the axial length and lenticular progressive myopia}

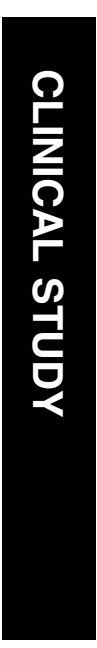

\begin{abstract}
Purpose To investigate the possible risk factors associated with lenticular progressive myopia and to compare the differences

risk factors predisposing to lenticular

progressive myopia.

Eye (2005) 19, 899-905. doi:10.1038/sj.eye.6701690;

published online 24 September 2004
\end{abstract} between patients with lenticular progressive myopias and senile cataracts.

Methods We retrospectively reviewed cases that had been diagnosed as lenticular progressive myopia with a discrete nuclear sclerotic cataract and progressive myopic changes in one hospital from January 1998 to February 2003. A total of 47 eyes of 35 patients were enrolled in this study. In all, 32 eyes of 29 cases of common senile cataract receiving cataract extraction surgery during the study period were randomly chosen (every four cases in time sequence within a 2-month period by two ophthalmologists' clinic in 2002) as the control group. We compared the preoperative refraction status, keratometry ( $K$-values) and axial lengths between these two groups. The possible ocular or systemic associating diseases were also investigated in the study group.

Results In the lenticular progressive myopia group, the mean age at surgery $(52.9 \pm 9.2$ years) is younger than that in the senile cataract group (68.1 \pm 7.3 years). The mean axial length in the study group $(25.68 \pm 1.93 \mathrm{~mm})$ is statistically significant longer than that in the control group $(22.97 \pm 0.83 \mathrm{~mm})(P<0.0001)$. Besides, patients with lenticular progressive myopia had significantly lower mean $K$-values (43.25 \pm 1.42 diopters) than patients with senile cataracts $(44.25 \pm 1.28$ diopters) $(P<0.01)$. There were no other ocular or systemic diseases closely associated with lenticular progressive myopia.

Conclusions Patients with nuclear cataract combined with lenticular progressive myopia have longer axial length than patients with senile cataract. The longer axial length may be one of the important
H-Y Lin ${ }^{1,2}$, C-W Chang ${ }^{3}$, H-Z Wang ${ }^{2}$ and R-K Tsai ${ }^{1,2}$

Keywords: lenticular progressive myopia; axial length; risk factor; nuclear cataract

\section{Introduction}

The clinical presentation of lenticular progressive myopia is well known. In 1946, Cordes $^{1}$ described a form of nuclear cataract in which a small central zone develops an increase in its refractive index resulting in lenticular progressive myopia. Cataract manifested by an opalescent nuclear sclerosis may result in a significant myopic refractive change as well as significant decrease in glare acuity and vision. ${ }^{2,3}$ Cataract extraction, including

phacoemulsification, allowed a satisfactory surgical result. Brown and Hill ${ }^{4}$ found that the myopic change precedes the development of the cataract in patients with lenticular progressive myopia.

However, the risk factors predisposing to lenticular progressive myopia have rarely been mentioned so far. The purpose of the present study was to compare the differences between cataracts with lenticular progressive myopia and age-related cataracts, and to assess the possible risk factors associated with lenticular progressive myopia.

\section{Materials and methods}

We retrospectively studied patients being diagnosed as lenticular progressive myopia with a discrete nuclear sclerotic cataract at two staffs' clinic in Kaohsiung Medical University Hospital in Taiwan from January 1998 to February 2003. The diagnostic criteria were:
${ }^{1}$ Department of Ophthalmology Buddhist Tzu-Chi Medical Center

Buddhist Tzu-Chi University Hualien, Taiwan

${ }^{2}$ Department of Ophthalmology Kaohsiung Medical University

Kaohsiung, Taiwan

${ }^{3}$ Department of Ophthalmology Chung Shan Medical University Hospital Taichung, Taiwan

Correspondence: R-K Tsai Department of Ophthalmology Buddhist Tzu-Chi Medical Center

707 Section 3

Chung-Yang Road Hualien 970, Taiwan Tel: + 88638561825 $\times 2223$

Fax: + 88638577161

E-mail: rktsai@

tzuchi.com.tw

Received: 19 February 2004 Accepted: 13 July 2004 Published online: 24 September 2004 
Table 1 Clinical details of patients with lenticular progressive myopia

\begin{tabular}{|c|c|c|c|c|c|c|c|c|c|c|c|c|c|}
\hline $\begin{array}{l}\text { Case } \\
\text { no. }\end{array}$ & Sex & Age & $\begin{array}{l}\text { Affected } \\
\text { eye }\end{array}$ & $\begin{array}{l}\text { Original } \\
\text { refraction }\end{array}$ & $\begin{array}{l}\text { Pre-OP manifest } \\
\text { refraction }\end{array}$ & $\begin{array}{l}\text { Post-OP manifest } \\
\text { refraction }\end{array}$ & K1 & K2 & Mean K & $\begin{array}{l}\text { Axial length } \\
(\mathrm{mm})\end{array}$ & $\begin{array}{c}\text { Pre-OP } \\
\text { BCVA }\end{array}$ & $\begin{array}{c}\text { Post-OP } \\
\text { BCVA }\end{array}$ & $O P^{\mathrm{a}}$ \\
\hline 1 & $\mathrm{~F}$ & 60 & OS & -3 & -12.0 cyl-0.25 140 & -2.25 cyl-2.0 170 & 45.3 & 44.3 & 44.8 & 23.11 & $20 / 200$ & $20 / 30$ & ECCE \\
\hline 2 & $\mathrm{M}$ & 44 & OD & -3.25 & -6.25 cyl-0.75 77 & -1.75 & 42.5 & 41.88 & 42.19 & 26.68 & $20 / 40$ & $20 / 20$ & Phaco \\
\hline 3 & $\mathrm{M}$ & 48 & OD & -5.25 cyl- -0.2566 & -14.75 cyl-2.0 97 & -2.25 cyl-1.0 180 & 43.25 & 42.7 & 42.975 & 26.94 & $20 / 200$ & $20 / 20$ & ECCE \\
\hline 4 & $\mathrm{M}$ & 54 & OS & + 1.5 cyl-4.0 170 & -6.25 cyl-3.75 170 & NA & 45.05 & 41.4 & 43.225 & 23.85 & $20 / 1000$ & NA & Phaco \\
\hline \multirow[t]{2}{*}{5} & M & 32 & OD & -7.5 & -10 & -2.5 cyl-0.75 180 & 44 & 42.65 & 43.325 & 29.83 & $20 / 100$ & $20 / 40$ & ECCE \\
\hline & & & OS & -7.75 & -9.5 & -3.25 cyl-0.75 140 & 44.9 & 42.8 & 43.85 & 29.39 & $20 / 100$ & $20 / 20$ & ECCE \\
\hline 6 & $\mathrm{~F}$ & 48 & OD & -3.5 & -6.0 cyl -0.7543 & -0.5 cyl-3.0 180 & 43.8 & 43.38 & 43.59 & 25.17 & $20 / 100$ & $20 / 30$ & ECCE \\
\hline \multirow[t]{2}{*}{7} & $\mathrm{M}$ & 42 & OD & -12.0 cyl-1.25 180 & -23.0 cyl-2.75 180 & -2.25 cyl-2.5 170 & 45.37 & 43.5 & 44.435 & 27.47 & $20 / 200$ & $20 / 20$ & ECCE \\
\hline & & & OS & -11.0 cyl-2.0 160 & -21.25 cyl-1.5 163 & -1.0 cyl-4.5 180 & 45 & 43.1 & 44.05 & 27.33 & $20 / 100$ & $20 / 20$ & ECCE \\
\hline \multirow[t]{2}{*}{8} & $\mathrm{M}$ & 47 & OD & -1.25 cyl-1.5 173 & -6.0 cyl-2.0 170 & +0.5 cyl-2.5 180 & 40 & 43.15 & 41.575 & 23.93 & $20 / 40$ & $20 / 30$ & ECCE \\
\hline & & & OS & -1.5 cyl-0.5 45 & -7.75 cyl-0.5 53 & +0.5 cyl-1.5 180 & 43.8 & 45 & 44.4 & 23.97 & $20 / 200$ & $20 / 25$ & ECCE \\
\hline 9 & $\mathrm{~F}$ & 51 & OS & +0.25 cyl -1.060 & -7.75 cyl-1.25 61 & -0.75 cyl-0.5 160 & 46 & 46.75 & 46.375 & 22.49 & $20 / 200$ & $20 / 20$ & ECCE \\
\hline 10 & $\mathrm{M}$ & 54 & OD & -4.25 cyl- 0.7568 & -11.75 cyl-0.75 62 & -2.5 & 44.75 & 44.5 & 44.625 & 25.84 & $20 / 1000$ & $20 / 25$ & Phaco \\
\hline \multirow[t]{2}{*}{11} & $\mathrm{M}$ & 53 & OD & -5.75 & -12.75 cyl -0.7590 & -1.25 cyl-1.5 180 & 41.25 & 41.12 & 41.185 & 28.38 & $20 / 400$ & $20 / 40$ & ECCE \\
\hline & & & OS & -6 & -8.25 & -1.5 cyl-0.5 25 & 41.63 & 40.32 & 40.975 & 28.25 & $20 / 200$ & $20 / 15$ & ECCE \\
\hline \multirow[t]{2}{*}{12} & $\mathrm{M}$ & 50 & OD & -2.75 cyl -0.590 & -5.25 cyl-0.25 115 & -1.25 cyl-1.0 180 & 42.5 & 42.12 & 42.31 & 24.03 & $20 / 50$ & $20 / 20$ & ECCE \\
\hline & & & OS & -1.75 & -5.50 cyl-0.25 171 & -1.5 cyl -0.7550 & 42.5 & 42.12 & 42.31 & 23.82 & $20 / 100$ & $20 / 20$ & ECCE \\
\hline 13 & $\mathrm{M}$ & 54 & OD & -4.75 cyl-2.0 170 & -18.5 cyl-3.5 170 & cyl-1.25 180 & 45.13 & 44 & 44.565 & 27.56 & $20 / 200$ & $20 / 20$ & Phaco \\
\hline \multirow[t]{2}{*}{14} & M & 79 & OD & -4.5 & $\begin{array}{c}-10.75 \text { cyl-0.75 } \\
160\end{array}$ & -2.5 cyl-0.75 180 & 44.12 & 44.5 & 44.31 & 25.85 & $20 / 40$ & $20 / 25$ & ECCE \\
\hline & & & OS & -4.5 & -8.25 cyl-0.25 23 & -2.75 cyl -0.570 & 43.5 & 44 & 43.75 & 26.22 & $20 / 100$ & $20 / 20$ & ECCE \\
\hline \multirow[t]{2}{*}{15} & $\mathrm{M}$ & 55 & OD & -1.25 & -5.0 cyl-0.5 110 & NA & 40.7 & 40.75 & 40.725 & 26.04 & $20 / 100$ & NA & - \\
\hline & & & OS & -0.5 cyl-0.5 130 & -5.5 & cyl-1.0 100 & 40.6 & 41.1 & 40.85 & 25.83 & $20 / 200$ & $20 / 40$ & Phaco \\
\hline 16 & $\mathrm{~F}$ & 46 & OD & $-5.50 \mathrm{cyl}-2.063$ & -12.75 cyl-2.5 65 & -2.0 cyl -2.0180 & 44.75 & 45.5 & 45.125 & 26.36 & $20 / 100$ & $20 / 25$ & ECCE \\
\hline 17 & $\mathrm{M}$ & 50 & OD & -1.75 cyl-1.0 20 & -8.0 cyl -0.7532 & -1.0 cyl-2.25 170 & 42.1 & 43 & 42.55 & 25.74 & $20 / 100$ & $20 / 20$ & ECCE \\
\hline 18 & $\mathrm{~F}$ & 62 & OD & -4.0 cyl-0.75 180 & -12.0 cyl-1.0 180 & -1.5 cyl-3.0 10 & 45.2 & 44.4 & 44.8 & 26.92 & $20 / 100$ & $20 / 20$ & ECCE \\
\hline \multirow[t]{2}{*}{19} & M & 58 & OD & -8.5 cyl-1.25 86 & -10.25 cyl-0.75 70 & -0.75 cyl -1.080 & 45 & 45.1 & 45.05 & 25.92 & $20 / 30$ & $20 / 20$ & Phaco \\
\hline & & & OS & -8.75 & -14.50 cyl-0.75 45 & -0.75 cyl-0.5 15 & 45.1 & 45.25 & 45.175 & 26.35 & $20 / 40$ & $20 / 20$ & Phaco \\
\hline \multirow[t]{2}{*}{20} & $\mathrm{~F}$ & 51 & OD & -8.25 & -11 & -1.5 cyl-1.75 122 & 44.1 & 43.9 & 44 & 27.6 & $20 / 50$ & $20 / 15$ & Phaco \\
\hline & & & OS & -7.75 & -11.75 cyl-0.75 135 & NA & 44.75 & 43.77 & 44.26 & 27.77 & $20 / 30$ & NA & - \\
\hline 21 & $\mathrm{~F}$ & 46 & OD & -4.25 cyl -0.580 & -11 & -3.75 & 45.12 & 45.5 & 45.31 & 24.68 & $20 / 400$ & $20 / 20$ & Phaco \\
\hline 22 & $\mathrm{~F}$ & 48 & OD & -8.25 & -12.5 cyl-0.25 15 & NA & 43 & 42.9 & 42.95 & 29.34 & $20 / 30$ & NA & - \\
\hline 23 & $\mathrm{~F}$ & 58 & OD & $-2.5 \mathrm{cyl}-0.7590$ & -11.0 cyl-1.5 90 & -2.0 cyl-0.75 175 & 45.35 & 44.37 & 44.86 & 24.56 & $20 / 200$ & $20 / 20$ & Phaco \\
\hline 24 & $\mathrm{~F}$ & 50 & OD & -3.0 cyl-1.0 90 & -11.25 cyl -0.7578 & NA & 42.63 & 42.63 & 42.63 & 27.51 & $20 / 200$ & NA & - \\
\hline 25 & $\mathrm{~F}$ & 64 & OD & -3.0 cyl-1.5 84 & -5.5 cyl-1.0 168 & +0.75 cyl-1.5 96 & 43 & 44.1 & 43.55 & 23.31 & $20 / 200$ & $20 / 30$ & Phaco \\
\hline 26 & $\mathrm{M}$ & 49 & OD & $+0.5 \mathrm{cyl}+0.5171$ & -3.75 cyl-2.5 107 & -0.25 cyl-0.75 103 & 42.12 & 41.75 & 41.935 & 24.07 & $20 / 50$ & $20 / 30$ & Phaco \\
\hline 27 & M & 68 & OD & $+1.0 \mathrm{cyl}+1.01$ & -2.5 cyl-3.0 62 & +1.0 cyl-1.75 60 & 43 & 41.5 & 42.25 & 25.57 & $20 / 40$ & $20 / 20$ & Phaco \\
\hline 28 & $\mathrm{~F}$ & 52 & OD & -0.75 cyl -0.555 & -7.25 cyl-1.0 65 & -2.5 cyl-1.25 21 & 43.87 & 42.87 & 43.37 & 23.74 & $20 / 50$ & $20 / 25$ & Phaco \\
\hline \multirow[t]{2}{*}{29} & $\mathrm{M}$ & 68 & OD & $+0.25 \mathrm{cyl}+0.5168$ & -20.5 & -1.0 cyl-1.5 80 & 42.25 & 41.75 & 42 & 23.25 & $20 / 1000$ & $20 / 50$ & Phaco \\
\hline & & & OS & $+0.5 \mathrm{cyl}+0.52$ & -2.75 cyl-2.0 79 & -1.25 cyl -0.561 & 42 & 41.25 & 41.625 & 23.49 & $20 / 70$ & $20 / 50$ & Phaco \\
\hline
\end{tabular}




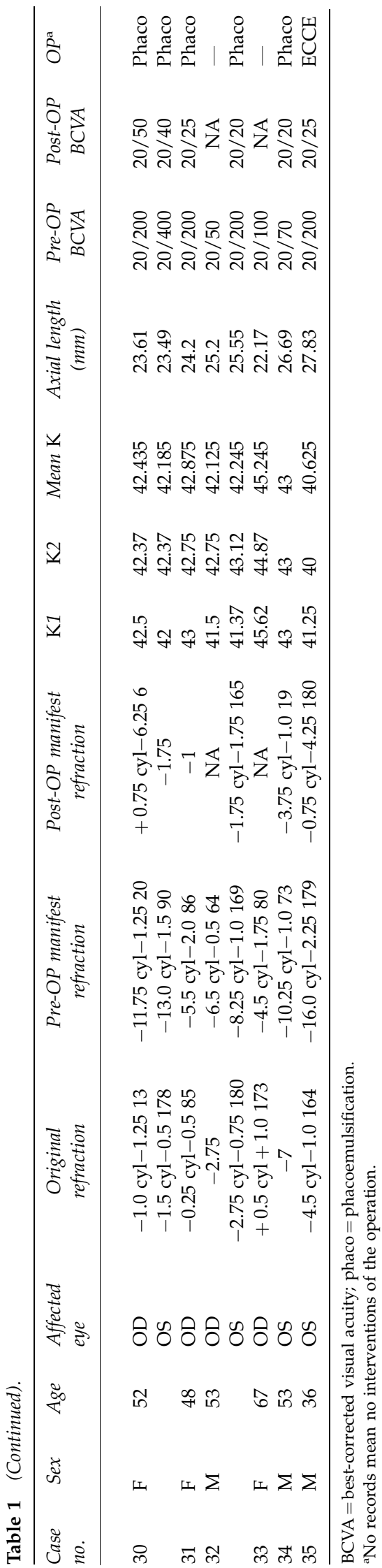

(1) progressive myopic shift starting at the age over 30 years old. (2) discrete nuclear sclerosis of lens without cortical and subcapsular opacities by slit-lamp biomicroscopy; and (3) loss of best-corrected visual acuity. Patients were excluded if they had no obvious histories or records of starting progressive myopic refractive changes after a stable refraction in adult age (older than 30 years), and without visually significant discrete nuclear sclerotic cataracts. Patients with progressive myopic change without stop since young age were also excluded. The refractive status 2-3 years before developing progressive lenticular myopia was recorded and compared with the refraction before the cataract surgery. For some patients, the original refractive status was unavailable, and the refraction of the old spectacles was used to represent the original refractive status. The details of 47 eyes of 35 patients consistent with our diagnostic criteria of lenticular progressive myopia were shown in Table 1. On the other hand, 32 eyes of 29 cases of ordinary age-related cataract receiving cataract extraction surgery were randomly chosen, every four cases being in time sequence within a 2-month period by two ophthalmologists' clinic in 2002, as a control group (Table 2). Age-related cataracts were defined as those presenting with nuclear sclerosis only or nuclear sclerosis combined with cortical opacity and/or subcapsular opacity in the senile age (over 55 years old). And there are no obvious myopic refractive changes in recent years. The sex distribution, age, manifest refractive status before and after the operation, and biometric data including the keratometry ( $K$-values) and the axial lengths in both groups were compared. The possible ocular or systemic associating diseases in patients with lenticular progressive myopia were also investigated. In 23 patients with only one eye affected with lenticular progressive myopia, the $K$-values and axial lengths were compared between the affected eyes and the fellow eyes. The axial length was recorded with an ultrasonic biometer (Model 820, Humphrey Instruments, USA), the $K$-value was measured with a keratometer manually (OM-4, Topcon, Tokyo, Japan), and the refractive status was measured with an autorefractor (RK-3000, Topcon, Tokyo, Japan). Cataracts were extracted by either extracapsular cataract extraction or phacoemulsification with IOL implantation. Biometric data in patients with lenticular progressive myopia and senile cataract were statistically analysed by Student's $t$-test.

\section{Results}

The mean age at the time of cataract extraction in the study group was $52.9 \pm 9.2$ years old, while that of the control group was $68.1 \pm 7.3$ years old. The sex distributions showed that male patients ( 30 eyes in 20 
Table 2 Clinical details of patients with senile cataract

\begin{tabular}{|c|c|c|c|c|c|c|c|c|c|}
\hline $\begin{array}{l}\text { Case } \\
\text { no. }\end{array}$ & Sex & Age & $\begin{array}{l}\text { Affected } \\
\text { eye }\end{array}$ & Pre-OP manifest refraction & K1 & K2 & Mean K & $\begin{array}{l}\text { Axial length, } \\
(\mathrm{mm})\end{array}$ & $O P$ \\
\hline 1 & M & 77 & OS & cyl + 1.5140 & 43.88 & 45.88 & 44.88 & 22.67 & ECCE \\
\hline 2 & $\mathrm{~F}$ & 76 & OD & -0.5 cyl -0.7590 & 46.3 & 46.75 & 46.525 & 21.65 & ECCE \\
\hline 3 & $\mathrm{~F}$ & 73 & OS & 0.75 & 42.8 & 43.5 & 43.15 & 22.45 & ECCE \\
\hline 4 & M & 81 & OD & 1.5 & 44.57 & 45.59 & 45.08 & 22.84 & ECCE \\
\hline 5 & M & 69 & OD & $+1.25 \mathrm{cyl}+3.0180$ & 45.9 & 47 & 46.45 & 21.68 & ECCE \\
\hline 6 & $\mathrm{~F}$ & 73 & OS & 2.25 & 43.13 & 43.23 & 43.18 & 22.83 & ECCE \\
\hline 7 & $\mathrm{~F}$ & 55 & OD & -3.0 cyl-1.5 90 & 44.23 & 43.45 & 43.84 & 22.69 & ECCE \\
\hline 8 & $\mathrm{~F}$ & 58 & OD & NA & 44.61 & 45.38 & 44.995 & 23.83 & Phaco \\
\hline 9 & $\mathrm{~F}$ & 64 & OS & $-5.0 \mathrm{cyl}-2.090$ & 45.4 & 45 & 45.2 & 23.83 & ECCE \\
\hline 10 & M & 71 & OD & +0.5 cyl-1.75 40 & 43.25 & 44.57 & 43.91 & 23.23 & ECCE \\
\hline 11 & M & 58 & OS & cyl +0.580 & 43.5 & 42.5 & 43 & 23.42 & Phaco \\
\hline \multirow[t]{2}{*}{12} & M & 69 & OD & -4.5 cyl-2.0 70 & 44.25 & 43.5 & 43.875 & 23.65 & ECCE \\
\hline & & & OS & -2.25 cyl-0.75 10 & 44.3 & 43.7 & 44 & 23.29 & ECCE \\
\hline 13 & $\mathrm{~F}$ & 63 & OD & $+1.0 \mathrm{cyl}+1.25180$ & 43.95 & 45.37 & 44.66 & 22.52 & ECCE \\
\hline \multirow[t]{2}{*}{14} & M & 68 & OD & 1 & 43.35 & 44.7 & 44.025 & 22.47 & ECCE \\
\hline & & & OS & +2.0 cyl + 1.590 & 42.75 & 44.67 & 43.71 & 22.53 & ECCE \\
\hline 15 & M & 61 & OS & +1.0 cyl-2.0 180 & 43.6 & 43.6 & 43.6 & 24.26 & ECCE \\
\hline 16 & $\mathrm{~F}$ & 76 & OS & $+1.5 \mathrm{cyl}+0.7540$ & 44.62 & 44.75 & 44.685 & 22.28 & Phaco \\
\hline \multirow[t]{2}{*}{17} & M & 84 & OD & 3.5 & 44.2 & 44.7 & 44.45 & 23.33 & Phaco \\
\hline & & & OS & 3.5 & 42.9 & 45.7 & 44.3 & 23.35 & Phaco \\
\hline 18 & $\mathrm{~F}$ & 68 & OD & $+1.25 \mathrm{cyl}+1.2540$ & 44.06 & 44.9 & 44.48 & 22.71 & ECCE \\
\hline 19 & $\mathrm{~F}$ & 74 & OS & $+2.5 \mathrm{cyl}+1.0180$ & 43.68 & 45.25 & 44.465 & 22.23 & ECCE \\
\hline 20 & M & 66 & OD & cyl-4.0 28 & 42.38 & 42.88 & 42.63 & 23.62 & ECCE \\
\hline 21 & M & 73 & OD & $+1.0 \mathrm{cyl}+0.75180$ & 44 & 44.75 & 44.375 & 22.79 & Phaco \\
\hline 22 & $\mathrm{~F}$ & 71 & OD & -2.5 cyl -0.570 & 46.75 & 46.75 & 46.75 & 21.12 & Phaco \\
\hline 23 & M & 59 & OS & 2 & 43.02 & 42.46 & 42.74 & 22.71 & Phaco \\
\hline 24 & M & 59 & OD & -2.5 cyl-1.25 80 & 41.91 & 42.43 & 42.17 & 24.87 & Phaco \\
\hline 25 & $\mathrm{~F}$ & 69 & OS & +0.5 cyl-1.0 30 & 47.1 & 47.35 & 47.225 & 21.93 & Phaco \\
\hline 26 & M & 65 & OS & $+0.5 \mathrm{cyl}+0.5180$ & 43.88 & 43.63 & 43.755 & 23.97 & Phaco \\
\hline 27 & $\mathrm{~F}$ & 66 & OD & $+1.75 \mathrm{cyl}+0.5160$ & 42.3 & 43.06 & 42.68 & 22.56 & ECCE \\
\hline 28 & $\mathrm{~F}$ & 59 & OD & $+0.5 \mathrm{cyl}+1.2590$ & 44.8 & 45.12 & 44.96 & 23.75 & Phaco \\
\hline 29 & $\mathrm{~F}$ & 69 & OD & 1 & 41.67 & 42.53 & 42.1 & 23.99 & ECCE \\
\hline
\end{tabular}

Phaco $=$ phacoemulsification.

Table 3 Sex distributions in different groups of cataracts

\begin{tabular}{lcc}
\hline & $\begin{array}{c}\text { Male } \\
\text { (eyes/patients) }\end{array}$ & $\begin{array}{c}\text { Female } \\
\text { (eyes/patients) }\end{array}$ \\
\hline Lenticular myopia & $30 / 20$ & $17 / 15$ \\
Senile cataract & $17 / 14$ & $15 / 15$ \\
\hline
\end{tabular}

patients) were more than female patients (17 eyes in 15 patients) in the study group, but were equal in the control group (Table 3 ).

The mean axial length was $25.68 \pm 1.93 \mathrm{~mm}$ in the study group, and $22.97 \pm 0.83 \mathrm{~mm}$ in the control group. It was statistically significant longer in the lenticular progressive myopia group $(P<0.0001$, Table 4$)$. Patients with lenticular progressive myopia also had significant lower mean $K$-values (43.25 \pm 1.42 diopters (D)) than patients with senile cataract $(44.25 \pm 1.28 \mathrm{D})(P<0.01$, Table 4). The original refractive status before starting myopic progression in the study group ranged from
Table 4 Mean $K$-value and axial length of all eyes

\begin{tabular}{lccl}
\hline & $\begin{array}{c}\text { Study group } \\
(\mathrm{N}=47)\end{array}$ & $\begin{array}{c}\text { Control group } \\
(\mathrm{N}=32)\end{array}$ & P-value* \\
\hline Mean K-value (D) & $43.25 \pm 1.42$ & $44.25 \pm 1.28$ & $<0.01$ \\
Mean axial length (mm) & $25.68 \pm 1.93$ & $22.97 \pm 0.83$ & $<0.0001$ \\
\hline $\mathrm{D}=$ diopter. & & & \\
${ }^{*}$ Unpaired $t$-test. & & & \\
& & &
\end{tabular}

hyperopia to myopia. There were five hyperopic eyes, and the mean refractive status before the change was $-3.947 \pm 3.343 \mathrm{D}$, the refraction at the time of cataract surgery was $-10.471 \pm 4.772 \mathrm{D}$ (Figure 1 ).

In the study group, there were 23 patients (65.7\%) with only one eye affected. We compared the data of $K$-values and axial lengths between the affected and the fellow eyes in these 23 patients. The mean $K$ was $43.63 \pm 1.37 \mathrm{D}$ in the affected eyes, and $43.76 \pm 2.07 \mathrm{D}$ in the fellow eyes. The mean axial length was $25.41 \pm 1.88 \mathrm{~mm}$ in affected eyes, and $25.0 \pm 1.87 \mathrm{~mm}$ in the unaffected. The data of 


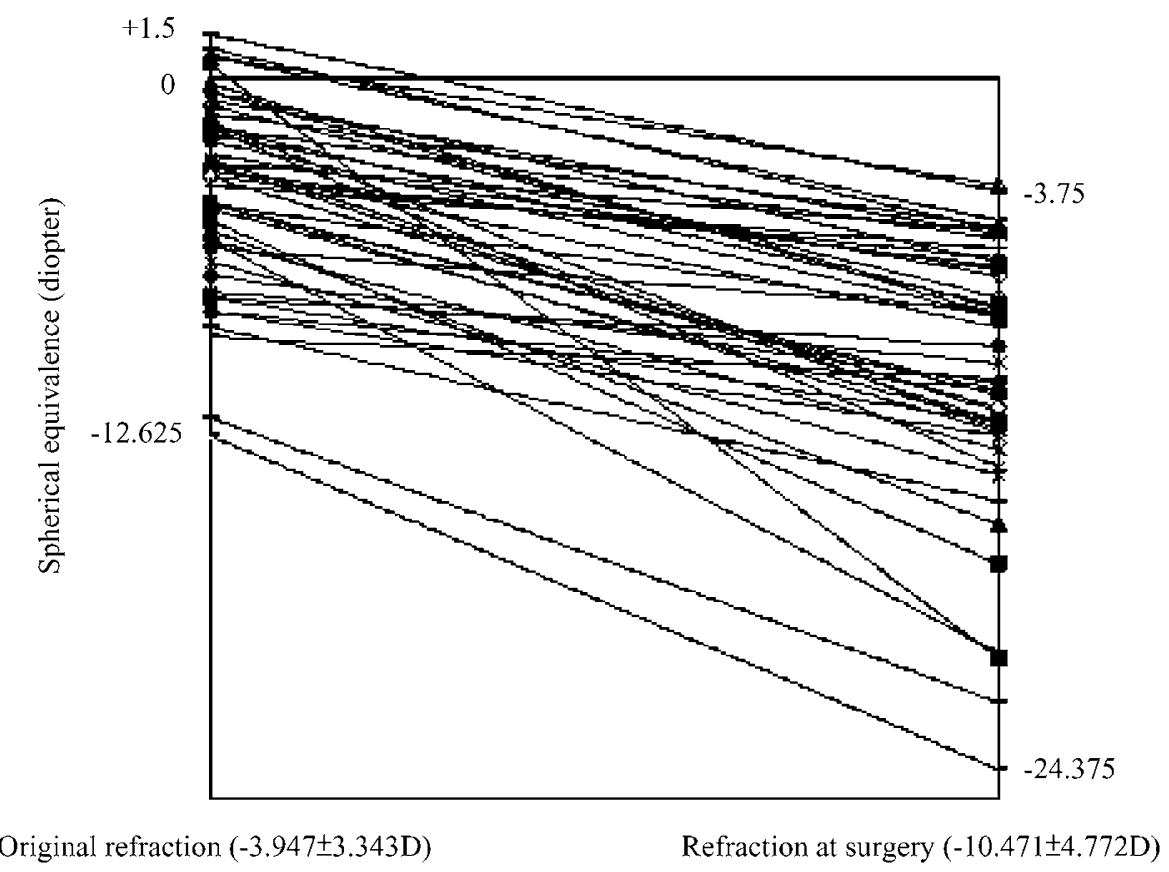

Figure 1 Refractive status changes between the time of starting the lenticular progressive myopia and the time at surgery. The duration of such changes was 2-3 years. There are five hyperopic eyes originally. The original mean refractive status before the change was $-3.947 \pm 3.343 \mathrm{D}$, and after the lenticular progressive change, became $-10.471 \pm 4.772 \mathrm{D}$.

Table 5 Mean $K$-value and axial length between affected and unaffected eyes in patients with only one eye with obvious lenticular myopia $(N=23)$

\begin{tabular}{lrrc}
\hline & Affected eyes & Unaffected eyes & P-value* \\
\hline Mean K-value (D) & $43.63 \pm 1.37$ & $43.76 \pm 2.07$ & 0.75 \\
Mean axial length (mm) & $25.41 \pm 1.88$ & $25.0 \pm 1.87$ & 0.47 \\
\hline
\end{tabular}

$\mathrm{D}=$ diopter. ${ }^{*}$ Unpaired $t$-test.

Table 6 Associated ocular and systemic diseases in patients with lenticular myopia

\begin{tabular}{llll}
\hline Ocular diseases & $\mathrm{n}$ & Systemic diseases & $\mathrm{n}$ \\
\hline Posner-Schlossman syndrome & 1 & Hypertension & 4 \\
Angle closure glaucoma & 1 & Hyperlipidaemia & 2 \\
Retinal detachment & 1 & Rheumatoid arthritis & 2 \\
Dry eye & 1 & Diabetes & 1 \\
Maculopathy & 1 & Hyperurecaemia & 1 \\
& & Ischaemic heart disease & 1 \\
& Thyroid disease & 1 \\
& Fatty liver & 1 \\
& Uterine myoma & 1 \\
& Appendicitis & 1 \\
& & Pituitary macroadenoma & 1 \\
\hline
\end{tabular}

$n=$ number of patients.

$K$-values and axial lengths both showed no significant differences between the affected and unaffected eyes (Table 5).
The associating ocular and systemic diseases in patients with lenticular progressive myopia were analysed (Table 6). A total of 16 patients were associated with certain systemic diseases, and five patients had cataracts combined with other ocular diseases. There were no specific ocular or systemic disorders closely associated with lenticular progressive myopia.

Excluding six eyes not receiving cataract extraction and one unavailable datum, 28 of 40 eyes (70\%) had bestcorrected visual acuity better than $20 / 25$ after the operation (Table 1).

\section{Discussion}

In 1987, Brown and Hill ${ }^{4}$ concluded that simple myopia does not appear to predispose to cataract formation. It is development of the cataract itself, particular in patients with nuclear sclerosis, which causes the refractive change towards myopia. The healthy ageing eye and eyes with cortical cataract or subcapsular cataract, but without nuclear sclerosis, continue to show a gradual hyperopic change with time. The myopic shift observed in nuclear cataract was independent of whether an eye started out as being refractively myopic or hyperopic. However, only the data of refraction instead of biometric data were investigated in their study. Although the definite refractive status before the beginning of the myopic shift was unavailable in some patients in our study, and the 
refractive data of old spectacles were used in these eyes, our results showed that they could range from hyperopia to myopia (there were five hyperopic eyes). Our study showed the same results as that of Brown and Hill ${ }^{4}$ that the original refraction was not the key factor to start the lenticular myopic shift. Since the refractive status was the result of nuclear sclerosis, we used the biometric data, including the $K$-value and axial length, and not only the refractive status, to study the possible risk factors associated with lenticular progressive myopia.

The patients enrolled in the present study had a special form of cataracts with visual significant lenticular progressive myopia, not cataracts accompanying axial myopia with or without notable induced myopic shift. To investigate the true lenticular progressive myopia, not the progressive 'axial' myopia, all the patients enrolled in this study had obvious histories of starting progressive myopic shift after the age of 30 years. The growth of axial length occurred in two stages in humans: an early or infantile stage, which Sorsby and co-workers assumed to conclude at age 3 years, and the subsequent juvenile stage, ending at the age of 14 years. The eye appears to have achieved its full growth by the age of 13 or 14 years. At the completion of ocular growth at age of 14 years, they found that the male eye had attained the average axial length of $24.1 \mathrm{~mm}$, and the female $23.7 \mathrm{~mm} .{ }^{5}$ Sorsby also suggested that in younger children in whom there was a greater increase in axial elongation there also could be noted a greater capacity for compensatory changes. This manifests itself in the generalization that the longer the eye, the deeper the anterior chamber, the flatter the cornea, and the less the lens refractive power. In our study, patients with lenticular progressive myopia had significant lower mean $K$-values and longer axial lengths than those with the senile cataract. This compensatory result was compatible with Sorsby's theory.

Nuclear sclerotic cataract as a cause of visual loss in young patients with axial myopia has been described in as early as $1980 .{ }^{6}$ De Natale $e t a l^{7}$ found that myopic eyes with axial myopias always had higher lens opacity values than that of emmetropic ones, and this result was statistically significant after the age of 20 years. Kaufman and Sugar $^{3}$ reviewed a series of young patients with myopia who had a visually disabling cataract. They concluded these patients are a select group that tends not to follow the established associations between the visually significant lens opacities, age, and the degree of myopia. Nuclear sclerotic cataract in young patients had also been described in Taiwan. ${ }^{8}$ Chen et al suggested that patients with an axial length greater than $27.0 \mathrm{~mm}$ had the greatest risk of developing lenticular progressive myopia. However, there was no control group in these studies. And in our clinical observation, not every patient with longer axial length (greater than $26 \mathrm{~mm}$ ) would develop lenticular progressive myopia. To clarify the differences between the lenticular progressive myopia and common cataract, we selected the age-related cataracts, which had no progressive myopic shift, to be the control group, and compared the data between these two groups to find out the possible risk factors of lenticular progressive myopia.

In our series, the mean age at cataract operation in the study group was $52.9 \pm 9.2$ years old, which was younger than the defined age of senile, 55 years old. In Taiwan, an epidemiological study indicated that the age-related cataracts were more common in women than in men. ${ }^{9}$ The sex distribution in senile cataracts was different from that in our patients with lenticular myopia. The male is more predominant than the female among patients with lenticular progressive myopia (30 eyes vs 17 eyes).

Another study by Chen showed the same result as ours in Taiwanese patients. ${ }^{7}$ The difference of mean age between study and control group is partly explained by the bias of criteria of case selection between two groups. The discrete nuclear sclerosis with lenticular progressive myopia in our study was a different form of cataract from the ordinary senile nuclear cataract. It occurred in younger patients, more in male gender, and belonged to the presenile cataract.

We are aware of the possibility of metabolic disorders, like galactosemia, causing the presenile cataract. An oxidative damage to the lens proteins may also cause the formation of large and dense molecular aggregates inducing a reversible lens myopia and nuclear cataract. ${ }^{10}$ The rapid development of nuclear cataract in patients under hyperbaric oxygen treatment strongly supports the oxidative theory of nuclear cataract formation. Nutrition deficiencies ${ }^{11}$ and ultraviolet radiation ${ }^{12}$ had been reported predisposing to cataract development. However, there was no close relationship between the associating ocular or systemic disorders and lenticular progressive myopia in the present study.

A significant difference of the axial length between the lenticular progressive myopia group and the senile cataract group means the long axial length was a unique association with lenticular progressive myopia in our study. Weale ${ }^{13}$ quoted Fisher's work ${ }^{14}$ on lens fibre stress to show that the latently accommodated lens of the hypermetrope would be relaxed, whereas, in the myopic eye, lens would be stressed by a continually unrelaxed zonnule. Fisher suggested that myopic lenses are subject to greater 'ciliary stress' than emmetropic lenses, primarily because of the decreased accommodative demand placed on myopic eyes. The theory failed to explain the high incidence (11 in 12 eyes) of unilateral cataract in Kaufman and Sugar's series of patients. ${ }^{3}$ However, in our series, 12 of 35 patients (34.3\%) were affected in both eyes. Lenticular progressive myopias 
happened in both eyes consecutively, but not simultaneously in every case. The longer axial length in lenticular progressive myopia caused fewer accommodative demand and greater fibre stress of lens at near working than the ordinary ones in senile cataract. This possibility may explain why the long axial length is one of the risk factors in some patients with lenticular progressive myopia, but not all. We still do not know why the starting time and the degree of progressive myopic shift are different in both eyes in some individuals. Besides axial length, there should be other unknown trigger factors to start the myopic shift.

The biometric data between the affected and unaffected eyes in 23 patients with unilateral lenticular progressive myopia showed no significant differences in both $K$-values and axial lengths. Whether the unaffected eyes will develop lenticular progressive myopia a few years later or not needs further observations and long-term studies. Our retrospective study has its limitation to answer the question.

In our study, many of the patients following surgery had myopic results. To reduce the postoperative anisometropic condition after one eye cataract extraction, most of the patients would like to choose the IOL with low myopic results in biometric examinations, especially for some patients having pre-existing myopia in both eyes. Besides, after discussing with patients, low myopic refraction following surgery made patients more convenient at near working. The visual acuity after cataract extraction is usually good.

Lenticular progressive myopia is a specific form of cataracts, and it is different from the senile cataract. It may develop in both eyes simultaneously or consecutively. Low myopias, emmetropias even hyperopias with long axial lengths can develop lenticular progressive myopias, and the refraction status is not the key factor to start its development. The longer axial length may be one of the important risk factors predisposing to lenticular progressive myopia. The detailed mechanism of developing lenticular progressive myopia is still unclear, and it needs further clarifying.

\section{Acknowledgement}

This study was supported by the research grant TCRD 93-08 from Buddhist Tzu Chi Medical Center.

\section{References}

1 Cordes FC. Cataract Types. American Academy of Ophthalmology and Otolaryngology, 1946, cited from ref. 2.

2 Levin ML. Opalescent nuclear cataract. J Cataract Refract Surg 1989; 15: 576-579.

3 Kaufman BJ, Sugar J. Discrete nuclear sclerosis in young patients with myopia. Arch Ophthalmol 1996; 114: 1178-1180.

4 Brown NAP, Hill AR. Cataract: the relation between myopia and cataract morphology. Br J Ophthalmol 1987; 71: 405-414.

5 Curtin BJ (ed). The development of refraction. The Myopias: Basic Science and Clinical Management. Harper \& Row Publishers Inc., New York, 1985, pp 33-36.

6 O'Donnell Jr FE, Maumenee AE. Unexplained' visual loss in axial myopia: cases caused by mild nuclear sclerotic cataract. Ophthalmic Surg 1980; 11: 99-101.

7 De Natale R, Romeo G, Fama F, Scullica L. Human lens transparence in high-myopic subjects. Ophthalmologica 1992; 205: 7-9.

8 Chen SN, Lin KK, Chao AN, Kuo YH, Ho JD. Nuclear sclerotic cataract in young patients in Taiwan. J Cataract Refract Surg 2003; 29: 983-988.

9 Tsai SY, Hsu WM, Cheng CY, Liu JH, Chou P. Epidemiologic study of age-related cataracts among an elderly Chinese population in Shih-Pai, Taiwan. Ophthalmology 2003; 110: 1089-1095.

10 Palmquist BM, Philipson B, Barr PO. Nuclear cataract and myopia during hyperbaric oxygen therapy. Br J Ophthalmol 1984; 68: 113-117.

11 Cunning RG, Mitchell P, Smith W. Diet and cataract. Ophthalmology 2000; 107: 450-456.

12 Michael R, Brismar H. Lens growth and protein density in the rat lens after in vivo exposure to ultraviolet radiation. Invest Ophthalmol Vis Sci 2001; 42: 402-408.

13 Weale R. A note on a possible relation between refraction and a disposition for senile nuclear cataract. Br J Ophthalmol 1980; 64: 311-314.

14 Fisher RF. Senile cataract: a comparative study between lens fibres stress and cuneiform opacity formation. Trans Ophthalmol Soc UK 1970; 90: 93-109. 